\title{
Lipophilic X-ray contrast agents diatrizoyl double esters with cholesterol and cholic acid
}

\author{
Pushap G. Singh, ${ }^{a}$ Jo Klaveness ${ }^{a}$, Frode Rise ${ }^{b}$, and Arne Jørgen Aasen ${ }^{a^{*}}$ \\ ${ }^{a}$ School of Pharmacy, University of Oslo, P. O. Box 1155, Blindern, N-0317 Oslo, Norway. ${ }^{b}$ \\ Department of Chemistry, University of Oslo, P. O. Box 1033, Blindern, N-0316 Oslo, Norway. \\ *Email: ajaasen@farmasi.uio.no
}

\section{Dedicated to Professor Kjell Undheim on the Occasion of his $70^{\text {th }}$.birthday (received 26 Apr 01; accepted 18 Oct 01; published on the web 26 Oct 01)}

\begin{abstract}
Potential biodegradable and organ-specific X-ray contrast agents have been prepared by linking diatrizoic acid to cholesterol and cholic acid, respectively, via a spacer group containing a carbonate double ester. In vitro experiments revealed that the cholic acid esters were enzymatically degraded in the presence of pig liver esterase, while the double esters with cholesterol appeared to be stable, possibly due to low solubility in water.
\end{abstract}

Keywords: X-Ray contrast agents, diatrizoic acid, cholesterol, cholic acid, double ester carbonates, pig liver esterase

\section{Introduction}

X-ray contrast agents for parenteral use today are triiodinated benzene derivatives with hydrophilic substituents to secure high water-solubility. These agents have similar pharmacokinetic properties such as extracellular distribution and renal elimination. ${ }^{1,2}$ During the past there has been considerable interest in organospecific contrast agents; e.g. agents for liver imaging. ${ }^{1,3}$

Various approaches have been evaluated to target contrast agents to the liver: liposome encapsulated water-soluble iodinated agents, ${ }^{4}$ iodinated fat emulsions, ${ }^{5}$ solid particles, ${ }^{6}$ and iodinated cholesterol derivatives. ${ }^{7-9}$ Long residual time in the liver and other organ systems is a major problem with these agents. 
Bile acid drug conjugates, e.g. the anticancer drug chlorambucil, ${ }^{10,11}$ are potential liverspecific targeting drugs. So-called double esters are frequently used in prodrug design because of their high affinity for unspecific esterases. ${ }^{12}$ The antibiotic pivampicillin is a typical example. ${ }^{13}$ Double esters have also been evaluated in particulate contrast agents. ${ }^{6}$

Chloroethoxycarbonyl chloride has been extensively used to prepare linkers in contrast agent such as gas generating polymers. ${ }^{14-16}$ The preparation of potential biodegradable conjugates between diatrizoic acid and the steroids cholesterol and cholic acid, respectively, and evaluation of their degradability in vitro, constitutes the subject of the present report.

\section{Results and Discussion}

\section{Synthesis}

Epimeric cholest-5-en-3 $\beta$-yl $1(R, S)$-chloroethyl carbonates (2) were prepared in quantitative yield by esterification of cholesterol (1) with $( \pm)$-1-chloroethoxycarbonyl chloride according to Dang et al., ${ }^{17}$ cf. Scheme 1. The epimeric double esters 3 were subsequently prepared in $66 \%$ yield by esterification of diatrizoic acid as its tetrabutylammonium salt with the chlorocarbonates 2 in DMF, in the presence of catalytic amounts of potassium iodide.

$t$-Butyl cholate (5) was prepared as described by Bonar-Law ${ }^{18}$ who first reacted cholic acid (4) with trifluoroacetic anhydride (TFAA) to the corresponding C-24 mixed anhydride and with the C-3 and C-7 hydroxyl groups trifluoromethyl acetylated, $c f$. Scheme 2. This mixed anhydride was then treated with $t$-butanol followed by hydrolysis of the C-3 and C-7 trifluoromethyl acetyl groups with aqueous ammonia furnishing $t$-butyl cholate (5).

The C-3 position of $t$-butyl cholate (5) was selectively ${ }^{19-21} O$-acylated with ( \pm )-1chloroethoxycarbonyl chloride to the chlorocarbonate esters 6 in $85 \%$ yield. Subsequent esterification of the chlorocarbonates 6 with tetrabutylammonium diatrizoate in DMF using potassium iodide as catalyst, yielded the $t$-butyl protected esters 7 in $79 \%$ yield. Deprotection with TFA furnished the epimeric cholic acid conjugates 8 in $95 \%$ yield. 

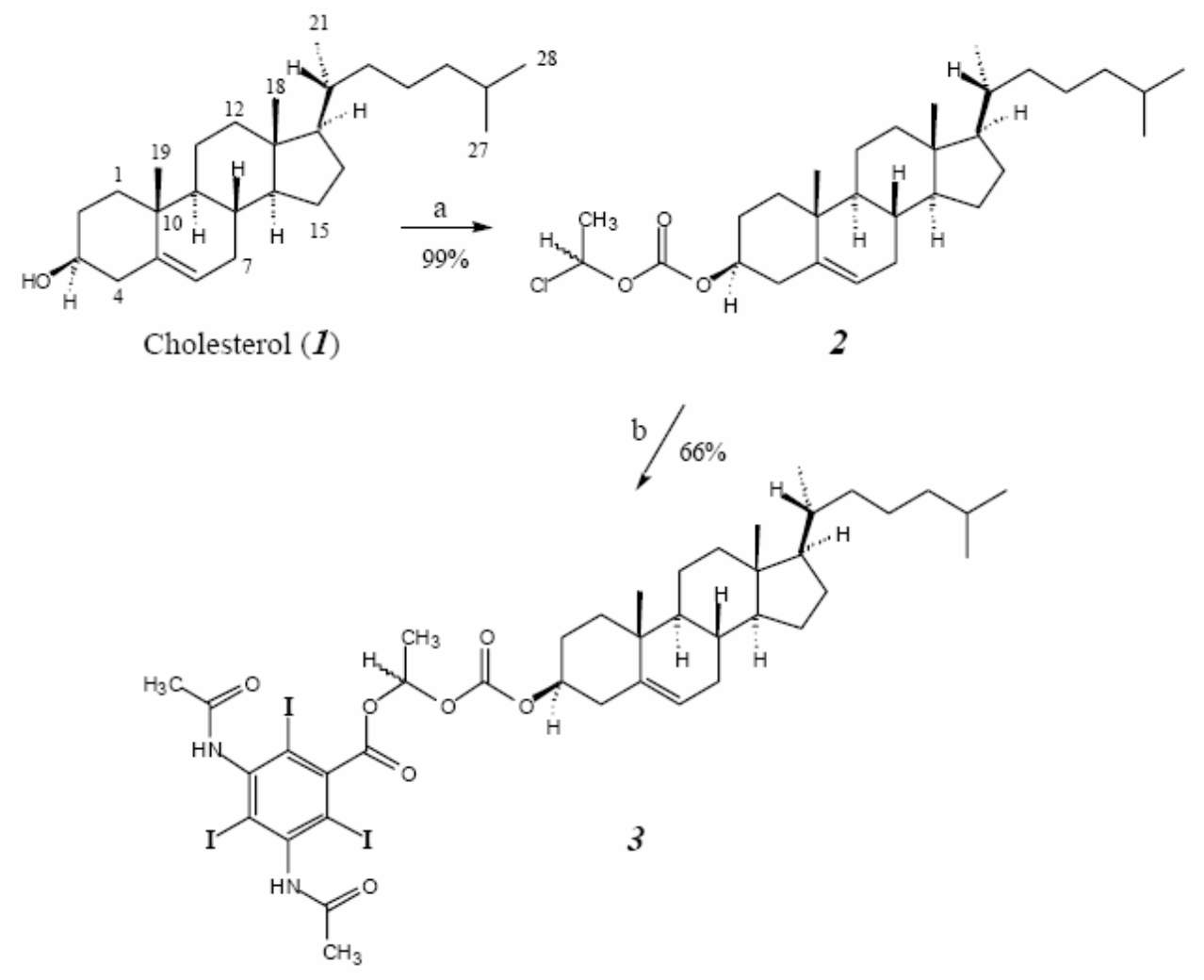

Scheme 1. a: ( \pm )-1-Chloroethoxycarbonyl chloride, pyridine, $\mathrm{CH}_{2} \mathrm{Cl}_{2} ; \mathbf{b}$ : Diatrizoic acid as $\mathrm{Bu}_{4} \mathrm{~N}^{+}$salt, KI, DMF 
Cholic acid (4)

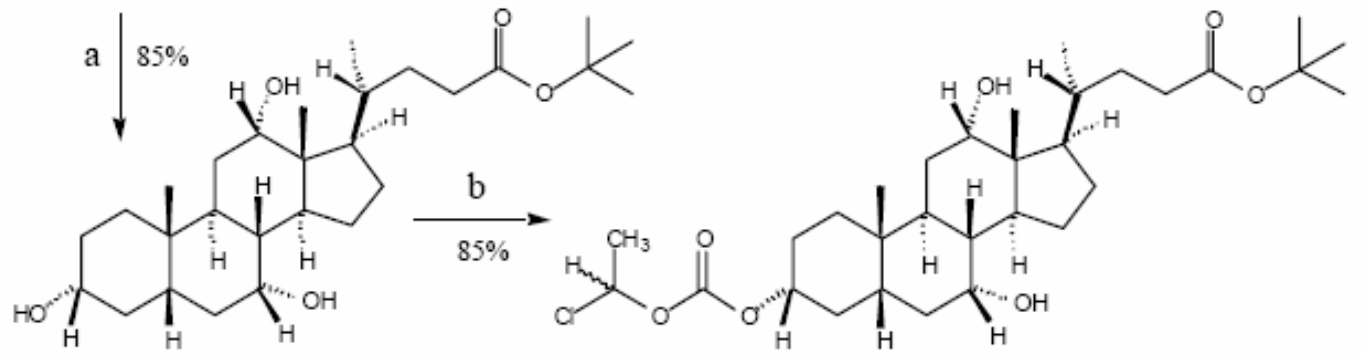

$t$-Butyl cholate (5)

6

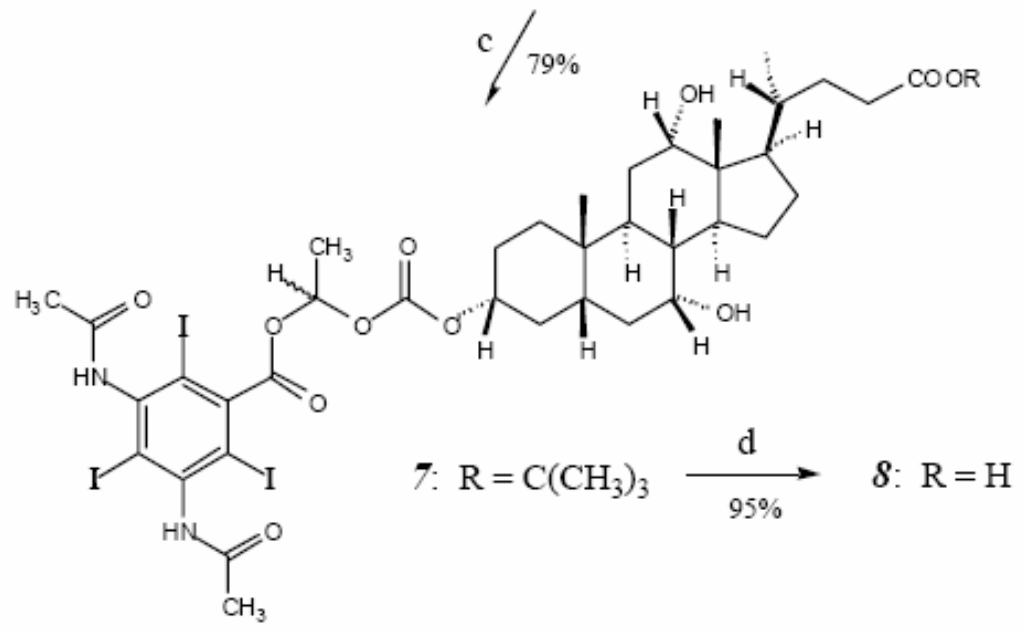

Scheme 2. a: (i) TFAA, (ii) $t-\mathrm{BuOH}$, (iii) $25 \% \mathrm{NH}_{3}$; b: ( $( \pm$ )-1-Chloroethoxycarbonyl chloride, DMAP, $\mathrm{CH}_{2} \mathrm{Cl}_{2} ;$ c: Diatrizoic acid as $\mathrm{Bu}_{4} \mathrm{~N}^{+}$salt, KI, DMF; d: TFA, $\mathrm{CH}_{2} \mathrm{Cl}_{2}$

\section{Enzymatic degradation}

Degradation studies with pig liver esterase revealed that the cholic acid conjugates $\mathbf{8}$ was a good substrate for the enzyme. The half life of the two epimeric esters $\mathbf{8}$ was found to be less than three hours.

The cholesterol derived double esters $\mathbf{3}$ appeared to be stable in similar degradation studies. The stability might be due to its low solubility in aqueous solutions. 


\section{Experimental Section}

General Procedures. ${ }^{1} \mathrm{H}$ NMR spectra were recorded at 200, 300, and $500 \mathrm{MHz}$ using Varian Gemini 200, Varian XL 300 (manual), and Bruker Avance DRX 500 spectrometers. ${ }^{13} \mathrm{C}$ NMR spectra were recorded at 50,75 and $125 \mathrm{MHz}$ using the above mentioned spectrometers. Assignments of NMR shifts have been limited to the diagnostically important parts, e.g. the contrast agent segment and the spacer (= the 1-chloro-ethoxycarbonate portion). Similarly, we report only assigned ${ }^{13} \mathrm{C}$ NMR shifts for selected parts of the molecules comprising the contrast agent segment, the spacer and the resonances of $\mathrm{C}_{3}, \mathrm{C}_{7}, \mathrm{C}_{12}, \mathrm{C}_{21}$ in the steroid moieties. Additional resolved resonances are listed without assignments. Presented assignments for ${ }^{1} \mathrm{H}$ and ${ }^{13} \mathrm{C}$ NMR spectra are based on published data for relevant compounds. ${ }^{22-24}$ Melting points were measured with a Reichert melting point microscope and are uncorrected. FAB and EI spectra were obtained using a Trio-2 Mass Spectrometer, VG Biotech Ltd. The EI spectra were recorded using 70 electronvolt ionizing voltage. Electrospray MS was obtained using a Bruker Apex 4.7 instrument. Elemental analyses were performed by Ilse Beetz, Kronach, Germany. TLC analyses were performed on silica gel plates (Merck 4500).

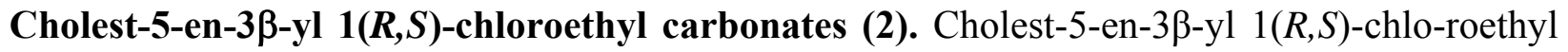
carbonates (2) were prepared in quantitative yield as described by Dang et al. ${ }^{17}$

Cholest-5-en-3 $\beta$-yl 1(R,S)-(3,5-diacetamido-2,4,6-triiodobenzoyloxy)ethyl carbonates (3). Diatrizoic acid tetrabutylammonium salt $(1.73 \mathrm{~g}, 2.0 \mathrm{mmol})$ was added to a solution of cholest-5-

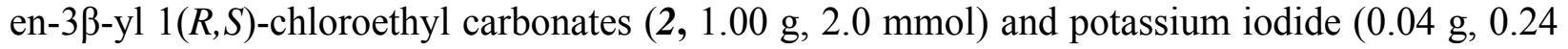
mmol) in DMF $(30 \mathrm{ml})$ and stirred for $3 \mathrm{~h}$ at $60{ }^{\circ} \mathrm{C}$. The mixture was cooled and diluted with $\mathrm{CHCl} 3(150 \mathrm{ml})$. The organic phase was washed with saturated aqueous $\mathrm{NaHCO}_{3}(4 \times 150 \mathrm{ml})$, water $(2 \times 150 \mathrm{ml})$ and dried over $\mathrm{Na}_{2} \mathrm{SO}_{4}$. The solvent was removed in vacuo and the product purified by flash chromatography on silica gel $\left(5 \% \mathrm{MeOH} / \mathrm{CHCl}_{3}\right)$ furnishing the carbonates 3 as a colourless solid $(1.41 \mathrm{~g}, 66 \%)$. Mp. $221-222{ }^{\circ} \mathrm{C}\left(\mathrm{dec}\right.$.); $\mathrm{R}_{\mathrm{f}} 0.38\left(5 \% \mathrm{MeOH} / \mathrm{CHCl}_{3}\right) ;{ }^{1} \mathrm{H}$ NMR $\left(500 \mathrm{MHz}, \mathrm{DMSO}-\mathrm{d}_{6}\right): \delta 0.64\left(3 \mathrm{H}, \mathrm{s}, \mathrm{C}-18\left(\mathrm{H}_{3}\right)\right), 0.83\left(6 \mathrm{H}, \mathrm{d}, \mathrm{C}-26 / 27\left(\mathrm{H}_{3}\right)\right), 0.88(3 \mathrm{H}, \mathrm{d}, \mathrm{C}-$ 21( $\left.\left.\mathrm{H}_{3}\right)\right), 0.96\left(3 \mathrm{H}, \mathrm{s}, \mathrm{C}-19\left(\mathrm{H}_{3}\right)\right), 1.64(3 \mathrm{H}, \mathrm{d}), 2.00\left(6 \mathrm{H}, \mathrm{s}, \mathrm{CH}_{3} \mathrm{CONH}-\right), 4.39(1 \mathrm{H}, \mathrm{m}), 5.35(1$ $\mathrm{H}, \mathrm{m}), 6.90(1 \mathrm{H}, \mathrm{m}), 10.04(2 \mathrm{H}, \mathrm{d}, \mathrm{NH}) ;{ }^{13} \mathrm{C}$ NMR $\left(125 \mathrm{MHz}, \mathrm{DMSO}-\mathrm{d}_{6}\right): \delta 12.3(\mathrm{C}-18), 19,2$ (C-21), 19,5 (C-19), 23.9 (C-39, C-41), 27.8 (C-30), 36,7 (C-10), 37.0 (C-1) 78.8 (C-3), 79.8 (C29), 97.0 (C-33, C-37), 109.7 (C-35), 123.1 (C-6), 139.8 (C-5), 145.4 (C-32), 146.2 (C34, C-36), 152.2 (C-28), 166.1 (C-38, C-40), 168.3 (C-31). Anal. found (C) 45.75, (N) 2.87, (H) 5.27; Calcd. for C41H57N2O7I3 (C) 46.00, (H) 5.37, (N) 2.62 .

$3 \alpha-[1(R, S)$-Chloroethyl carbonate] $t$-butyl $7 \propto 12 \alpha$-dihydroxy-5 $\beta$-cholan-24-oates (6). ( \pm )1-Chloroethoxycarbonyl chloride $(0.40 \mathrm{~g}, 2.8 \mathrm{mmol})$ was added dropwise to a cooled $\left(0{ }^{\circ} \mathrm{C}\right)$ 
solution of $t$-butyl $3 \alpha_{2} 7 \alpha, 12 \alpha$-trihydroxy-5 $\beta$-cholan-24-oate (5) (= $t$-butyl cholate ${ }^{18}, 1.00 \mathrm{~g}, 2.2$ mmol) and 4- $N, N$-dimethylaminopyridine $(0.36 \mathrm{~g}, 2.9 \mathrm{mmol})$ in $\mathrm{CH}_{2} \mathrm{Cl}_{2}(15 \mathrm{ml})$. The mixture was stirred for $24 \mathrm{~h}$ at room temperature and refluxed for $20 \mathrm{~min}$. The solution was cooled, diluted with $\mathrm{CH}_{2} \mathrm{Cl}_{2}(100 \mathrm{ml})$, and washed with saturated aqueous $\mathrm{CuSO}_{4}(100 \mathrm{ml} \times 4)$, saturated aqueous $\mathrm{NaHCO}_{3}\left(100 \mathrm{ml}\right.$ x 2), and brine $(100 \mathrm{ml})$. The organic phase was dried over $\mathrm{MgSO}_{4}$. The solvent was removed under reduced pressure and the product purified by column chromatography on alumina (grade $1,0.5 \% \mathrm{MeOH} / \mathrm{CHCl}_{3}$ ) giving the carbonates 6 as a solid (1.04 g, 85\%). Mp. $76-80{ }^{\circ} \mathrm{C} ; \mathrm{R}_{\mathrm{f}} 0.66\left(5 \% \mathrm{MeOH} / \mathrm{CHCl}_{3}\right) ;{ }^{1} \mathrm{H} \mathrm{NMR}\left(300 \mathrm{MHz}, \mathrm{CD}_{2} \mathrm{Cl}_{2}\right): \delta 0.67$ $\left(3 \mathrm{H}, \mathrm{s}, \mathrm{C}-18\left(\mathrm{H}_{3}\right)\right), 0.92\left(3 \mathrm{H}, \mathrm{s}, \mathrm{C}-19\left(\mathrm{H}_{3}\right)\right), 0.96\left(3 \mathrm{H}, \mathrm{s}, \mathrm{C}-21\left(\mathrm{H}_{3}\right)\right), 1.42(9 \mathrm{H}, \mathrm{s}), 1.69(3 \mathrm{H}, \mathrm{d}$, $5.73 \mathrm{~Hz}), 3.84(1 \mathrm{H}, \mathrm{m}, \mathrm{C}-7(\mathrm{H})), 3.98(1 \mathrm{H}, \mathrm{m}, \mathrm{C}-12(\mathrm{H})), 4.47(1 \mathrm{H}, \mathrm{m}, \mathrm{C}-3(\mathrm{H})), 6.30$ (1 H, q, $6.49 \mathrm{~Hz}) ;{ }^{13} \mathrm{C}$ NMR $\left(75 \mathrm{MHz}, \mathrm{CD}_{2} \mathrm{Cl}_{2}\right): \delta 12.6(\mathrm{C}-18), 17.5$ (C-21), 22.5 (C-19), $23.5(\mathrm{C}-15)$, 25.5 (C31), 26.9 (C-9), 28.2 (C-16, C-26, C-27, C-28), 28.5 (C-11) 31.3 (C-2, C-23), 32.8 (C22), 35.0 (C-6, C-10), 35.6 (C-1, C-20), 39.8 (C-4, C-8), 41.6 (C-5), 42.2 (C-14), 46.8 (C13), 47.6 (C17), 68.5 (C-7), 73.3 (C-12), 80.0 (C-25, C-30), 84.9 (C-3) 154.4 (C-29), 174.6 (C-24). Anal. found (C) 65.24, (H) 9.34; Calcd. for $\mathrm{C}_{31} \mathrm{H}_{51} \mathrm{O}_{7} \mathrm{Cl}(\mathrm{C})$ 65.19, (H) 9.00.

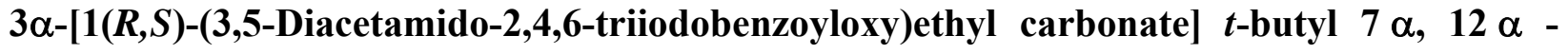
dihydroxy-5 $\beta$-cholan-24-oates (7). Diatrizoic acid tetrabutylammonium salt ( $0.99 \mathrm{~g}, 1.2 \mathrm{mmol})$ was added to a solution of $3 \alpha-[1(R, S)$-chloroethyl carbonate] $t$-butyl $7 \alpha, 12 \alpha$-dihydroxy-5 $\beta$ cholan-24-oates $(6,0.87 \mathrm{~g}, 1.4 \mathrm{mmol})$ and $\mathrm{KI}(0.02 \mathrm{~g}, 0.12 \mathrm{mmol})$ in DMF $(20 \mathrm{ml})$. The mixture was kept at $60{ }^{\circ} \mathrm{C}$ for $3 \mathrm{~h}$, cooled, and diluted with $\mathrm{CHCl}_{3}(100 \mathrm{ml})$. The $\mathrm{CHCl}_{3}$-solution was washed with saturated aqueous $\mathrm{NaHCO}_{3}(4 \times 100 \mathrm{ml})$ and water $(2 \times 100 \mathrm{ml})$. The organic phase was dried over $\mathrm{Na}_{2} \mathrm{SO} 4$ and concentrated to dryness in vacuo. The product was purified by column chromatography on alumina (grade 1, 3\% $\mathrm{MeOH} / \mathrm{CHCl} 3$ ) furnishing the carbonates 7 as a solid (0.88 g, 79\%). Mp. 196-201 ${ }^{\circ} \mathrm{C}$ (dec.); $\mathrm{R}_{\mathrm{f}} 0.15$ (5\% MeOH/CHCl 3$) ;{ }^{1} \mathrm{H} \mathrm{NMR} \mathrm{(200} \mathrm{MHz,}$ $\left.\left.\mathrm{CD}_{2} \mathrm{Cl}_{2}\right): \delta 0.66\left(3 \mathrm{H}, \mathrm{s}, \mathrm{C}-18\left(\mathrm{H}_{3}\right)\right), 0.89(3 \mathrm{H}, \mathrm{s}), \mathrm{C}-19\left(\mathrm{H}_{3}\right)\right), 1.13\left(3 \mathrm{H}, \mathrm{d}, \mathrm{C}-21\left(\mathrm{H}_{3}\right)\right), 1.42(9 \mathrm{H}$, s), $2.17(3 \mathrm{H}, \mathrm{s}),, 3.81(1 \mathrm{H}, \mathrm{m}, \mathrm{C}-7(\mathrm{H})), 3.95$ (1 H, m, C-12(H)), $4.43(1 \mathrm{H}, \mathrm{m}, \mathrm{C}-3(\mathrm{H})), 7.34$ (1 $\mathrm{H}, \mathrm{m}), 8.85$ (2 H, m, NH) ${ }^{13} \mathrm{C}$ NMR (50 MHz, DMSO-d $): \delta 23.8$ (C-40, C-42), 27.3 (C31), 28.7 (C-26, C-27, C-28), 68.8 (C-7), 73.6 (C-12), 80.5 (C-3), 94.0 (C-30), 96.4 (C34, C-38), 108,6 (C-36), 145.0 (C-37), 145.1 (C-35), 147.1 (C-33), 153.2 (C-29), 167.2 (C-32), 170.4 (C41); MS (FAB): m/z $1171[\mathrm{M}+\mathrm{Na}]^{+}, 1153[\mathrm{M}+\mathrm{Na}-\mathrm{H} 2 \mathrm{O}]^{+}, 1045[\mathrm{M}+\mathrm{Na}+\mathrm{H}-\mathrm{I}]^{+}$. Anal. found (C) 43.75, (H) 5.22, (N) 2.55; Calcd. for $\mathrm{C}_{42} \mathrm{H}_{59} \mathrm{~N}_{2} \mathrm{O}_{11} \mathrm{I}_{3}(\mathrm{C})$ 43.92, (H) 5.18, (N) 2.44.

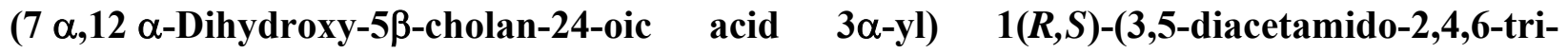
iodobenzoyloxy)ethyl carbonates $(8)$. TFA $(1.5 \mathrm{ml})$ was added to a cooled solution $\left(0{ }^{\circ} \mathrm{C}\right)$ of $3 \alpha$-[1(R,S)-(3,5-diacetamido-2,4,6-triiodobenzoyloxy)ethyl carbonate] t-butyl $\mathbf{7 \alpha}, \quad 12 \alpha$ dihydroxy-5 $\beta$-cholan-24-oates $(7,0.20 \mathrm{~g}, 0.18 \mathrm{mmol})$ in $\mathrm{CH}_{2} \mathrm{Cl}_{2}(5 \mathrm{ml})$. The solution was stirred for $45 \mathrm{~min}$ at room temperature, diluted with ethyl acetate $(50 \mathrm{ml})$, and washed with water $(3 \mathrm{x}$ 
$50 \mathrm{ml})$. The organic phase was dried over $\mathrm{Na}_{2} \mathrm{SO}_{4}$, concentrated to dryness in vacuo, and the product 7 purified by flash chromatography on silica gel $\left(20 \% \mathrm{MeOH} / \mathrm{CHCl}_{3}\right)$. The colourless product $(0.18 \mathrm{~g}, 95 \%)$ decomposed at $217-220{ }^{\circ} \mathrm{C}$; Rf $0.42\left(20 \% \mathrm{MeOH} / \mathrm{CHCl}_{3}\right) ;{ }^{1} \mathrm{H}$ NMR $(500$ MHz, DMSO-d 6 ): $\delta 0.59\left(3 \mathrm{H}, \mathrm{s}, \mathrm{C}-18\left(\mathrm{H}_{3}\right)\right), 0.86\left(3 \mathrm{H}, \mathrm{s}, \mathrm{C}-19\left(\mathrm{H}_{3}\right)\right), 0.92(3 \mathrm{H}, \mathrm{d}, 7.39 \mathrm{~Hz}, \mathrm{C}-$ 21( $\left.\left.\mathrm{H}_{3}\right)\right), 1.62(3 \mathrm{H}, \mathrm{d}, 4.99 \mathrm{~Hz}), 2.02\left(6 \mathrm{H}, \mathrm{s}, \mathrm{CH}_{3} \mathrm{CONH}-\right), 3.81(1 \mathrm{H}, \mathrm{m}, \mathrm{C}-12(\mathrm{H})), 4.23(1 \mathrm{H}, \mathrm{m}$, C-7(H)), $4.44(1 \mathrm{H}, \mathrm{m}, \mathrm{C}-3(\mathrm{H})), 6.9(1 \mathrm{H}, \mathrm{q}, 3.16 \mathrm{~Hz}), 10.13(2 \mathrm{H}, \mathrm{d}) ;{ }^{13} \mathrm{C}$ NMR $(125 \mathrm{MHz}$, DMSO-d $_{6}$ ): $\delta 21.1$ (C-36, C-38), 28.2 (C-27), 68.2 (C-7), 72.7 (C-12), 81.2 (C-3, C-26), 98.5 (C30, C-34), 110.9 (C32), 147.1 (C-31, C-33), 153.6 (C-29), 159.8 (C-25), 167.4 (C-28, C-35, C37); MS (electrospray; $\mathrm{MeOH} / \mathrm{H}_{2} \mathrm{O}$ ) m/z: $1093.0635[\mathrm{M}+\mathrm{H}]^{+}$; calcd. for $\mathrm{C}_{38} \mathrm{H}_{52} \mathrm{~N}_{2} \mathrm{O}_{11} \mathrm{I}_{3}$ : 1093.0699.

Stability of the diatrizoyl double esters 3 and 8 in esterase solutions. The conjugates $8(0.10$ g, $0.09 \mathrm{mmol})$ were added to a solution of pig liver esterase $(0.8 \mathrm{ml}$, Sigma E 3128) in ammonium carbonate buffer $(500 \mathrm{ml})$. The solution was shaken at $37{ }^{\circ} \mathrm{C}$ for $12 \mathrm{~h}$, and the reaction monitored by TLC. TLC revealed that the major part of the double ester (7) hydrolyzed during the first $3 \mathrm{~h}$. No conjugates could be detected after $12 \mathrm{~h}$. The mixture was freeze dried, and the residue purified by flash chromatography (silica gel, $\mathrm{CHCl}_{3}: \mathrm{MeOH}=4: 1$ ). Cholic acid and diatrizoic acid were identified by ${ }^{1} \mathrm{H}$ NMR and TLC.

The yield of cholic acid was $98 \%$. Hydrolysis was not observed in the absence of esterase.

The cholesterol derived double esters 3 did not hydrolyze when treated under similar conditions. 


\section{References and Notes}

1. Sovak, M. Radiocontrast Agents, Sovak, M. Ed., Springer Verlag: Berlin, 1984, p 1.

2. Stolberg, H. O.; McClennan, B. L. Curr. Prob. Diagn. Radiol. 1991, 20, 47.

3. Ferrucci, J. T.; Stark, D. D. Liver Imaging. Current Trends and New Techniques, Andover Medical Publishers: Boston, 1990.

4. Seltzer, S. E. Radiology 1989, 171, 19.

5. Ivancev, K.; Lunderquist, A.; Isaksson, A.; Hochbergs, P.; Wretlind, A. Acta Radiol. 1989, 30,449 .

6. Gjöen, T.; Holtz, E.; Strande, P.; Klaveness, J.; Leander, P.; Berg, A. Chapter 45 in reference 3 .

7. Longino, M. A.; Weichert, J. P.; Schwendner, S. W.; Szabo, S. M.; Counsell, R. E.; Glazer, G. M. Invest Radiol. 1983, 18, 275.

8. Counsell, R. E.; Seevers, R. H.; Korn, N.; Schwendner, S. W. J. Med. Chem. 1981, $24,5$.

9. Seevers, R. H.; Groziak, M. P.; Weichert, J. P.; Schwendner, S. W.; Szabo, S. M.; Longino, M. A.; Counsell, R. E. J. Med. Chem. 1982, 25, 1500.

10. Wess, G.; Kramer, W.; Schubert, G.; Enhsen, A.; Baringhaus, K.-H.; Glombik, H.; Müllner, S.; Bock, K.; Kleine, H.; John, M.; Neckermann, G.; Hoffmann, A. Tetrahedron Lett. 1993, $34,819$.

11. Kramer, W.; Wess, G.; Schubert, G.; Bickel, M.; Girbig, F.; Gutjahr, U.; Kowalewski, S.; Baringhaus, K.-H.; Enhsen, A.; Glombik, H.; Müllner, S.; Neckermann, G.; Schulz, S.; Petzinger, E. J. Biol. Chem. 1992, 267, 18598.

12. Bundgaard, H. Ed. Design of Prodrugs; Elsevier: Amsterdam, 1985.

13. Daehne, W.; v., Frederiksen, E.; Gundersen, E.; Lund, F.; Mørch, P.; Petersen, H. J.; Roholt, K.; Tybring, L.; Godtfredsen, W. O. J. Med. Chem. 1970, 13, 607.

14. Kan, Z. X.; Ivancev, K.; Hagerstrand, I.; Chuang, V. P.; Lunderquist, A. Acta Radiologica 1989, 30, 419.

15. Ivancev, K.; Lunderquist, A.; McCuskey, R.; McCuskey, P.; Wretlind, A. Acta Radiologica 1989, 30, 407.

16. Ivancev, K.; Lunderquist, A.; McCuskey, R.; McCuskey, P.; Wretlind, A. Acta Radiologica 1989, 30, 291.

17. Dang, V. A.; Olofson, R. A.; Wolf, P. R.; Piteau, M. D.; Senet, J.-P. G. J. Org. Chem. 1990, $55,1847$.

18. Bonar-Law, R. P.; Davis, A. P.; Sanders, J. K. M. J. Chem. Soc., Perkin. Trans. 1 1990, 2245.

19. Borsche, W. Ber. 1924, 57, 1620. 
20. Fieser, L. F.; Rajagopalan, S. J. Am. Chem. Soc. 1949, 71, 3935.

21. Fieser, L. F.; Rajagopalan, S. J. Am. Chem. Soc. 1950, 72, 5530.

22. Hatono, S. W. S.; Yazaki, A.; Yoshida, S. European Patent Application EPO 232 788, 1987.

23. Pretsch, E.; Clerc, T.; Seibl, J.; Simon, W. Tables of Spectral Data for Structure Determination of Organic Compounds, Eds. Fresenius, W.; Huber, J. F. K.; Pungor, E.; Rechnitz, G. A.; Simon, W.; West, T. S., 2nd. Edn. Springer-Verlag: Berlin, 1989.

24. Blunt, J. W.; Stothers, J. B. Org. Magn. Res. 1977, 9, 439. 